\title{
No evidence for selective follicle abortion underlying primary sex ratio adjustment in pigeons
}

\author{
Vivian C. Goerlich • Cor Dijkstra • Ton G. G. Groothuis
}

Received: 15 April 2009/Revised: 10 September 2009/Accepted: 20 October 2009/Published online: 14 November 2009

(C) The Author(s) 2009. This article is published with open access at Springerlink.com

\begin{abstract}
Primary sex ratio adjustment in birds has been extensively studied, yet the underlying physiological mechanisms are far from understood. Avian females are the heterogametic sex $(\mathrm{ZW})$, and the future sex of the offspring is determined at chromosome segregation during meiosis I, shortly before the oocyte is ovulated. Assuming that the mother can detect the sex of the developing oocyte before ovulation, it has been suggested that a follicle of the un-preferred sex could selectively be induced to become atretic and regress instead of being ovulated (selective follicle abortion). This potential mechanism has been proposed to underlie biased primary sex ratios in birds, including the homing pigeon (Columba livia domestica), which produces a modal clutch size of two eggs. However, without replacement by an additional, already mature follicle, abortion of a preovulatory follicle would most likely result in either reduced clutch sizes or laying gaps, since a not-yet-recruited follicle still needed to undergo the whole maturation phase. In the current study we killed female pigeons, which were adjusting embryo sex of first eggs according to change in body mass. We examined ovaries for signs of follicle abortion but did not find any supporting evidence. All females produced one or two mature follicles but only two out of the 56 experimental birds produced an additional third mature follicle. Therefore, our results do not corroborate the hypothesis that pigeon mothers manipulate primary offspring sex by selectively aborting follicles of the un-preferred sex.
\end{abstract}

Communicated by J. Graves

V. C. Goerlich $(\bowtie) \cdot$ C. Dijkstra · T. G. G. Groothuis Behavioural Biology, University of Groningen, Kerklaan 30,

9751NN Haren, The Netherlands

e-mail: V.Goerlich@rug.nl
Keywords Avian · Primary sex ratio $\cdot$ Mechanism . Oogenesis $\cdot$ Atresia $\cdot$ Meiotic drive $\cdot$ Columbidae

\section{Introduction}

It is well established that birds adjust their primary offspring sex ratio according to certain environmental circumstances, and several functional hypotheses have been put forward, proposing that higher fitness return parents would gain from an overproduction of either sons or daughters. Still, unraveling the proximate mechanisms underlying manipulation of primary offspring sex ratio is an ongoing quest (Krackow 1995; Pike and Petrie 2003; Alonso-Alvarez 2006). Acquiring more knowledge on this phenomenon is crucial for understanding its evolution and trade-offs. Recent research strongly suggests that maternal body condition and hormones play an important role in primary offspring sex ratio manipulation (reviewed by Pike and Petrie 2003; Alonso-Alvarez 2006). However, the pathways by which hormones might exert their effects on embryo sex determination remain speculative.

In birds, males are the homogametic sex (ZZ), while females produce gametes holding either of the sex chromosomes $\mathrm{Z}$ or $\mathrm{W}$. Which one of the chromosomes is retained in the developing ovum is determined during completion of meiosis I, shortly before the ovarian follicle ovulates the oocyte into the infundibulum of the oviduct (Johnson 2000; Rutkowska and Badyaev 2008). In order to produce the desired sex at fertilization, two earlier proposed mechanisms, both enabling manipulation of oocyte sex before ovulation, are potential candidates: Chromosome segregation during meiosis might not be random but skewed in a certain direction to retain the preferred sex chromosome in the ovum, while the other chromosome is 
displaced into the inactive polar body (meiotic drive; Howe 1977; Krackow 1995; Komdeur et al. 2002; Correa et al. 2005; Rutkowska and Badyaev 2008). Alternatively, in case of random meiosis, a mature follicle bearing the un-preferred sex could in theory be resorbed before ovulation, thereby giving way for the next follicle in the ovarian hierarchy (follicle abortion; Emlen 1997; Pike 2005). An important assumption underlying this model is that, in order to decide if the oocyte carries the preferred sex, the maternal physiological system can detect the sex of the oocyte after meiosis but before ovulation (and actual fertilization). In mammal literature the communication between the mother and gametes is a well-recognized concept (Georgiou et al. 2007; Fazeli 2008). It is likely that in birds as well the gamete undergoes changes at the molecular level after meiosis (Rutkowska and Badyaev 2008) and signals it's future sex to the mother.

Although no precise predictions on the nature of follicle abortion exist, we assume the process to be comparable to atresia, which is the degeneration and resorption of follicles (Johnson 2000). The ovary contains millions of undifferentiated small follicles from which the majority terminates development, becomes atretic, and is resorbed (Gilbert et al. 1983). Only some follicles will be selected to enter the phase of rapid yolk deposition and eventually reach the mature state of a preovulatory follicle. At ovulation the follicle releases the ovum into the oviduct and degenerates thereafter. The remaining follicular walls (postovulatory follicle) are visible for at least several days (Johnson 2000). However, a structure similar to a postovulatory follicle might also be the result of bursting atresia. In this case the follicle ruptures and releases its yolk content into the ovarian stroma, a process thought to occur also among more mature follicles (Romanoff and Romanoff 1949; Johnson 2000).

Follicles that first enter the phase of rapid yolk deposition suppress the development of following follicles to a certain extent, thereby building a visible hierarchy in size and development of preovulatory follicles (Johnson 2000). Consequently, abortion of a mature follicle just before ovulation will most likely result in delayed clutch initiation (if the first follicle would be aborted) or laying gaps (in case of an intermediate follicle in the size hierarchy) since the follicle following the aborted one would lag behind in development. The thereby prolonged egg intervals might prove detrimental to the parents and/or offspring because of increased vulnerability toward predators due to longer incubation periods and enlarged hatching asynchrony (Emlen 1997). An alternative strategy to avoid detrimental effects of laying gaps between eggs would be to restrict sex biases to first eggs only (Emlen 1997), a pattern shown in several experiments on homing pigeons (Columba livia; Pike 2005; Goerlich et al. 2009; Goerlich et al. unpublished data; Müller et al. unpublished data).
Columbidae are considered to be determinate layers with a modal clutch size of two eggs. Even if eggs are removed, pigeons do not produce additional replacement eggs, unlike several other bird species (e.g., Beukeboom et al. 1988; Blockstein 1989; Nager et al. 2001). It is therefore widely assumed that in pigeons per ovarian cycle only two follicles are recruited into the phase of rapid yolk deposition (Haywood 1993), which lasts approximately 6.5 days $(\sim 156 \mathrm{~h})$ in domestic homing pigeons $(C$. livia; Birrenkott et al. 1988; Fig. 1a). Following yolk completion, the mature follicles are ovulated with an interval of $44 \mathrm{~h}$ (Fig. 1a) into the oviduct, where subsequently fertilization and albumen and shell formation take place (Levi 1945). Anatomical studies on ovaries of ring doves (Streptopelia risoria) and band-tailed pigeons (Columba fasciata) did not reveal more than two mature follicles present prior to egg laying (Cuthbert 1945; March and Sadleir 1970; Gutierrez et al. 1975). However, Bartelmez (1912) states that in domestic pigeons occasionally three follicles entered the phase of rapid yolk deposition. Whether pigeons develop more than two follicles has important implications for the possibility of follicle abortion in itself. If only two follicles are produced, abortion would result in reduced clutch size of one egg only or prolonged egg intervals because a replacement follicle recruited only after abortion would need to undergo the whole maturation period of 6.5 days. Both phenomena do not occur frequently, if at all, in pigeons producing biased sex ratios (Pike 2005; Goerlich et al. 2009; Müller et al. unpublished data). Recruiting a third follicle a priori, as part of the regular ovarian hierarchy, could prevent these negative effects. The third follicle could act as replacement if the female was to manipulate offspring sex by aborting a follicle of the undesired sex.

Our aim was to explore the potential for follicle abortion in homing pigeons, and to do so we needed to induce females to bias their primary sex ratio. We therefore designed this study as a repeat of a previous experimental protocol and implanted females with testosterone to induce an overproduction of male embryos (Goerlich et al. 2009). However, females used in the current study underwent drastic changes in body mass, which possibly overruled the effects of the hormonal treatment. Females adjusted the primary sex ratio of first eggs not in response to testosterone but according to change in body mass (Goerlich et al. unpublished data). Still, this provided us with the opportunity to investigate whether follicle abortion is the mechanism underlying the manipulation of primary sex ratio. To do so we killed females after oviposition of the first egg and measured ovarian and follicle development. Depending on the number of follicles reaching mature state per ovulation cycle and potential follicle abortion, we predicted different scenarios being visible at the time of 

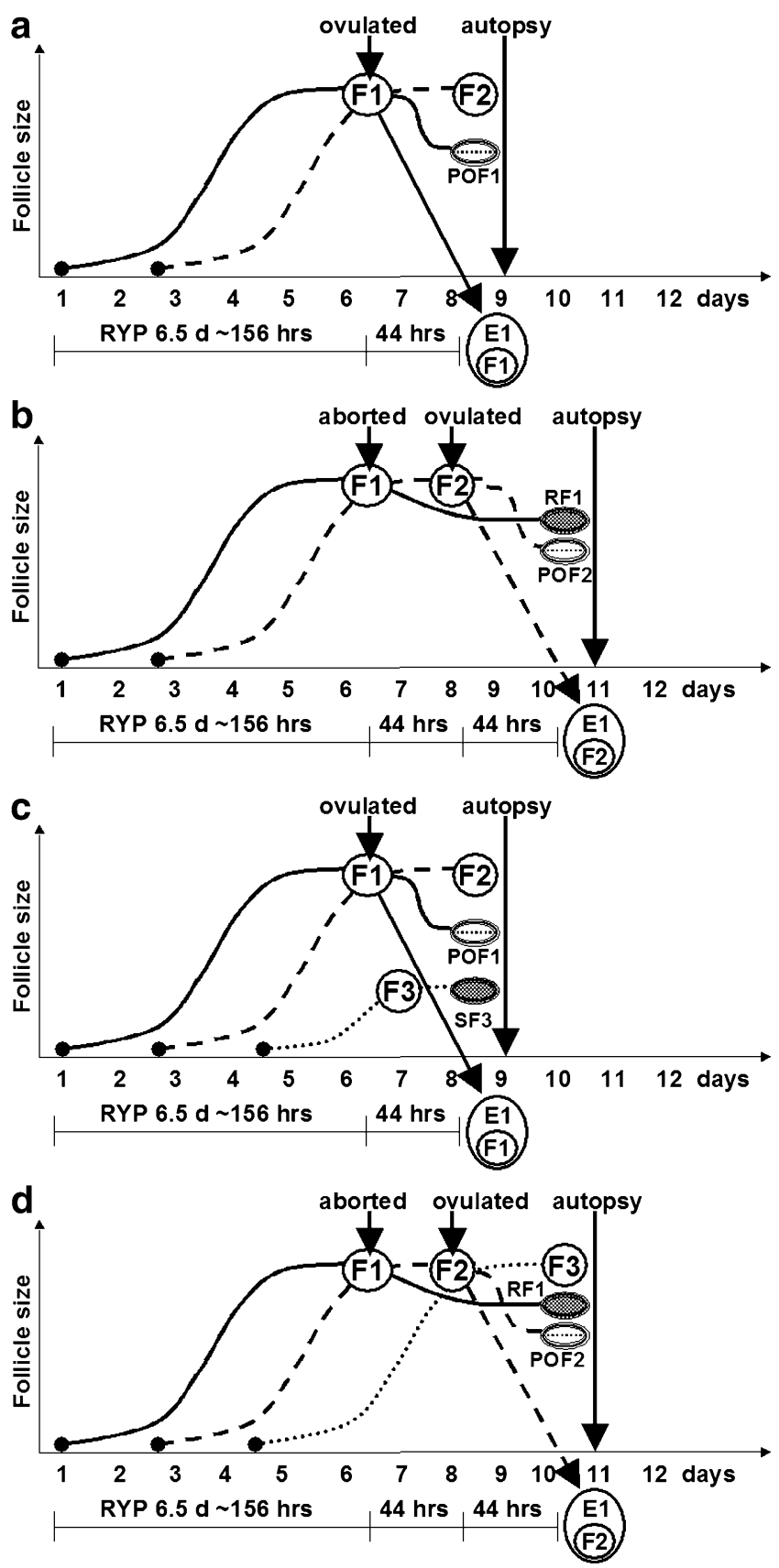

autopsy, which we schematically present in Fig. 1a-d. Figure 1a represents the most parsimonious scenario of two developing follicles without the option of selective follicle abortion. Figure 1a, b assumes that, per reproductive event, only two follicles enter the phase of rapid yolk deposition; Fig. 1c, d considers the possibility for the recruitment of an additional follicle. In case of meiosis resulting in the preferred sex (expected in $50 \%$ of the cases, based on random chance), the first follicle is ovulated to be laid as the first egg (Fig. 1a, c). In case of bearing the un-preferred sex, the first follicle would be aborted, thereby giving way to the follicle next in the hierarchy (Fig. 1b, d).
Fig. 1 a-d Schematic representation of potential follicle growth and abortion patterns in the homing pigeon. The sigmoid curves represent hypothetical growth dynamics of the subsequent follicles ( $F 1$ and $F 2$ ) and, after ovulation/abortion, the hypothetical course of regression. a Only two follicles enter the phase of rapid yolk deposition $(R Y P)$. The first follicle $(F I)$ is of the preferred sex, is ovulated, and laid as the first egg $(E 1)$. At the time of autopsy the ovary contains the mature second follicle $(F 2)$ and one postovulatory follicle sac (POF1). b Only two follicles enter the phase of rapid yolk deposition $(R Y P)$. The first follicle $(F 1)$ is of the un-preferred sex and is aborted. The second follicle $(F 2)$ is ovulated and laid as the first egg $(E 1)$ instead. No second egg is produced. At the time of autopsy, the ovary contains one regressed follicle $(R F 1)$ and one postovulatory follicle sac $(P O F 2)$. c More than two follicles enter the phase of rapid yolk deposition $(R Y P)$. The first follicle $(F 1)$ is of the preferred sex, is ovulated, and laid as the first egg $(E 1)$. The ovulation suppresses further development of the third follicle $(F 3)$. At the time of autopsy the ovary contains the mature second follicle $(F 2)$, one postovulatory follicle sac $(P O F 1)$, and one suppressed follicle (SF3). d More than two follicles enter the phase of rapid yolk deposition $(R Y P)$. The first follicle $(F 1)$ is of the un-preferred sex and is aborted. The second follicle $(F 2)$ is ovulated and laid as the first egg $(E 1)$ instead. At the time of autopsy the ovary contains the mature third follicle $(F 3)$, one regressed follicle $(R F 1)$ and one postovulatory follicle sac $(P O F 2)$

By performing the autopsies after oviposition of the first egg, we aimed at making inferences on the likelihood of these potential scenarios based on the number of mature, preovulatory follicles, postovulatory follicles, and possibly aborted (regressed) follicles.

\section{Materials and methods}

\section{Experimental design}

The experiment was conducted between September and December 2006. In total we used 60 pairs of homing pigeons, split over two successive blocks of 30 pairs each. Breeding pairs were housed indoors in single cages under a 14-h light cycle at $22^{\circ} \mathrm{C}$ room temperature. Birds had auditory and some visual contact with other pairs. Female age ranged from 1 to 6 years; thus all individuals were sexually mature.

To explore follicle abortion as the mechanism underlying embryo sex biases in pigeons, we needed to expose females to a treatment which causes the overproduction of one sex. We chose to implant females with testosterone, which had resulted in significant male-biased primary sex ratio in first, but not second eggs in our previous study (Goerlich et al. 2009).

First, all pairs produced an un-manipulated trial clutch to ensure proper reproductive activity (Cuthbert 1945) and to provide an intra-female control for plasma parameters and primary sex ratio (Goerlich et al. unpublished data). After all pairs had completed their first clutch, they were separated, and males were brought to outside unisex 
aviaries. Females received subcutaneous Silastic implants filled with crystalline testosterone, or empty sham implants (controls; Goerlich et al. 2009, unpublished data). Treatment alternated over the cages, and females were distributed evenly across the treatment groups based on age. We waited 3 days to have the implants effectively elevate female plasma testosterone levels and then re-introduced the males.

During both clutches, we checked nests for eggs once per hour during lights-on. Directly after oviposition of the first egg of the second clutch we brought the female to another room where she was immediately killed with $\mathrm{CO}_{2}$. After overnight storage at $10^{\circ} \mathrm{C}$ we weighed the birds $(0.1 \mathrm{~g})$, removed the reproductive organs, and stored these in saline at $10^{\circ} \mathrm{C}$ for several hours until taking of the measurements.

Freshly collected eggs were incubated at $38^{\circ} \mathrm{C}$ for $72 \mathrm{~h}$ to allow embryo sex determination (Goerlich et al. 2009, unpublished data).

From 37 females we collected a fertile first egg during both the trial and the implanted clutch and divided the change in sex of first eggs in four categories: first clutch daughter $\rightarrow$ second clutch son; first daughter $\rightarrow$ second daughter; first son $\rightarrow$ second son; first son $\rightarrow$ second daughter. In this way our analyses were sensitive to inter-female differences in baseline sex ratio. We then applied multinomial regressions with change in maternal body mass from the first to the second clutch as predicting variable and the four compositions as unordered categorical dependants, with first son $\rightarrow$ second daughter as reference category (Goerlich et al. unpublished data).

In this group of females in which embryo sex of first eggs was available from both experimental clutches the change in maternal body mass significantly predicted the change in composition of first egg sex. Females that gained mass more likely switched from a daughter to a son compared to females that lost mass (Goerlich et al. unpublished data). This gave us the opportunity to analyze the ovaries of these females for signs of follicle abortion as a hypothetical mechanism underlying the adjustment embryo sex in the eggs produced. From the remaining 19 females either the egg during the first or the second clutch did not develop an embryo. We could therefore not apply the same analysis to show whether these females were adjusting embryo sex as well and as a consequence excluded them from the group of females in which we found an overall relationship between change in body mass and change in first egg sex.

\section{Ovarian measurements}

Since we killed the females after laying the first egg, the remaining postovulatory follicle of this first ovulation event should be visible in the ovary of all females. However, no reliable data exist on how fast aborted (atretic) follicles might be eliminated. We expected to find regressed atretic follicles, clearly smaller than mature second follicles (see Fig. 1b, d). Alternatively, if aborted follicles would rupture and release their yolk, such follicles would resemble additional empty postovulatory follicles (Romanoff and Romanoff 1949; Johnson 2000). We therefore carefully examined the ovaries for postovulatory follicles (remnants of ovulated follicles) and signs of follicle atresia or remaining structures of aborted and subsequently regressed follicles. We also weighed the ovaries (without the larger follicles $(>6 \mathrm{~mm})$ ) to the nearest $0.001 \mathrm{~g}$ to explore potential outcomes in case the sex of the first egg resulted from aborting a follicle bearing the un-preferred sex: Ovaries might be heavier due to a higher amount of small follicles (in anticipation of repeated abortion) or the surplus remnants of regressed follicles. Alternatively, ovaries might be lighter due to repeated follicle resorption. To further explore these possible scenarios we also counted all small yolky follicles, which had not yet entered the phase of rapid yolk deposition (2.5 to $6.0 \mathrm{~mm}$ diameter). All larger maturing follicles were counted and weighed $(0.001 \mathrm{~g})$, and the diameter was measured using a digital caliper $(0.01 \mathrm{~mm})$. Three of the large follicles were damaged during the autopsy and could therefore not be measured.

All experimental procedures were carried out under approval of the animal experimentation committee of the University of Groningen (license DEC 4347D).

Statistical analyses

Two females had lost their implants, and two did not produce any eggs; therefore we excluded these birds from all further analyses.

We did not include all experimental females in our statistical analyses since we did not have complete data sets from the whole experimental group to explore the relation between change in body mass and change in first egg sex. Nevertheless, we chose to graphically present the data from all birds to provide a more comprehensive impression on follicle morphology in pigeons. To test whether follicle abortion underlies sex ratio manipulation, we incorporated only complete clutches from 37 females in which we could, on a group level, statistically confirm the change in embryo sex of first eggs according to change in maternal body mass (Goerlich et al. unpublished data).

We fitted general linear mixed models (GLMM) using MlwiN 2.02, with two hierarchical levels, female ID nested in block, to control for our experimental design. In separate models we tested the following dependant variables: ovarian weight (log transformed to achieve normal distribution), mass and diameter of the largest $(>15 \mathrm{~mm}$ diameter) 
follicle, and amount of small yolky follicles (2.5 to $6 \mathrm{~mm}$ diameter). Our models contained the following predictors: change in maternal mass between clutch 1 and 2 (the strongest predictor of primary sex ratio) and maternal mass at oviposition during clutch 2 , since the mass of reproductive organs might be related to the actual body mass of the individual. Furthermore, we incorporated the sex of the first egg laid in clutch 2, which might have resulted from follicle abortion and latency to oviposition (days between pairing and first egg, clutch 2) as predicting variables. Latency to oviposition might reflect the time needed to produce additional follicles after potential abortion.

Estimation procedure was specified as restricted iterative generalized least squares. In all tests, significance levels were set to an alpha $<0.05$ (two-tailed). We started with full models, including all predictors, and then sequentially excluded the term with the largest $p$ value following the backward stepwise method (Grafen and Hails 2002). Significance values are stated before the term was removed from the model.

Due to the small number of females bearing more than two mature follicles after having laid the first egg, no statistical tests were applicable to explore the potential factors explaining the occurrence of a third follicle.

\section{Results}

The ovarian follicles could clearly be assigned to two distinct size classes of small yolky ( $<6 \mathrm{~mm}$ diameter) and mature preovulatory follicles $(>15 \mathrm{~mm}$ diameter).This pattern was similar between the group of females in which we showed an adjustment of embryo sex $(N=37)$ and further 19 females (Fig. 2). In case of abortion resulting in yolk loss due to follicle rupture (Johnson 2000), we expected follicles of substantial lighter mass than usual for a certain diameter, but in all females the follicles were firm and showed a clear size-mass relationship following an exponential growth curve (Fig. 3).

We could not detect evidence supporting the occurrence of follicle abortion in the group in which the availability of two fertile first eggs enabled us to show an overall effect of change in body mass on sex of first eggs.

Out of these 37 females, two had not developed a second mature follicle, thus would have produced a single egg clutch. All the remaining 35 females developed two mature follicles, but none produced more than two. Thus, at the time of autopsy the ovary of 35 females contained one postovulatory and one mature preovulatory follicle (Fig. 1a), whereas two contained only one postovulatory follicle. We did not establish any regressed follicles or more than one postovulatory follicle.

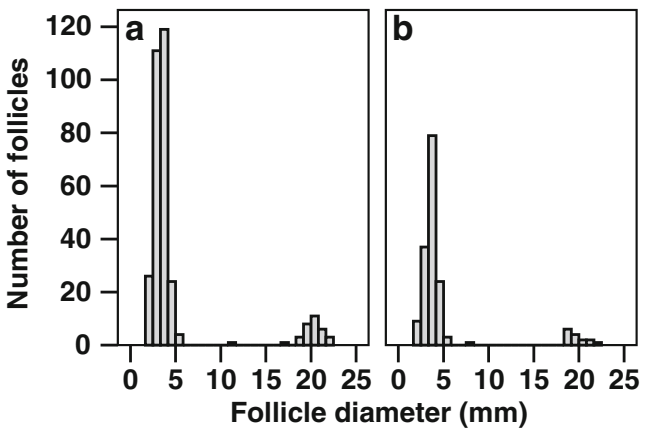

Fig. 2 a Frequency distribution of follicle diameters $(\mathrm{mm})$ in the group of females from which we determined both embryo sexes (clutch 1 and 2,N=37) and $\mathbf{b}$ the rest of the experimental females in which we could determine one embryo sex (clutch 1 or $2, N=19$ )

Neither ovarian mass, follicle diameter and mass, nor the amount of small yolky follicles was significantly predicted by change in maternal body mass (predicting change in sex ratio), absolute maternal body mass, first egg sex, or latency to oviposition (Table 1). However, follicle mass and diameter were, though, not significantly, to be positively associated with absolute maternal body mass (Table 1).

Regarding the ovarian morphology of the other 19 reproductively active females, from which we were not able to determine embryo sex during both clutches, we recorded four aberrant cases: Two females had developed a third large follicle, since after oviposition of the first egg the ovaries still contained two large yolky follicles $(>18.8 \mathrm{~mm}$ diameter, i.e., the second and third of the preovulatory hierarchy, Fig. 3). In both cases only one

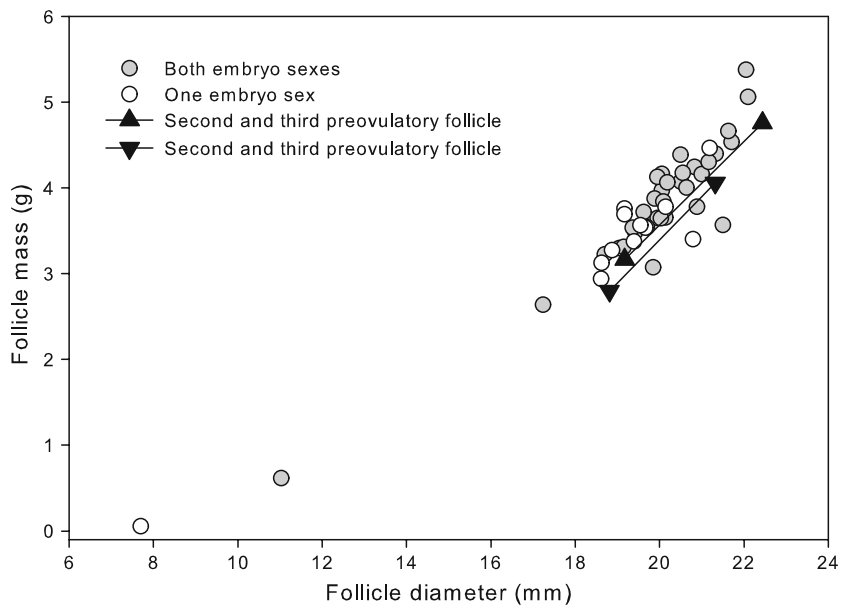

Fig. 3 Scatter plot of follicle mass by follicle diameter including preovulatory follicles larger than $6 \mathrm{~mm}$ of the group of females from which we determined both embryo sexes (gray circles, $N=37$ ) and the rest of the experimental females in which we could determine one embryo sex (white circles, black triangles, $N=19$ ). Circles represent single preovulatory follicles; triangles represent the second and third preovulatory follicles present in two females 


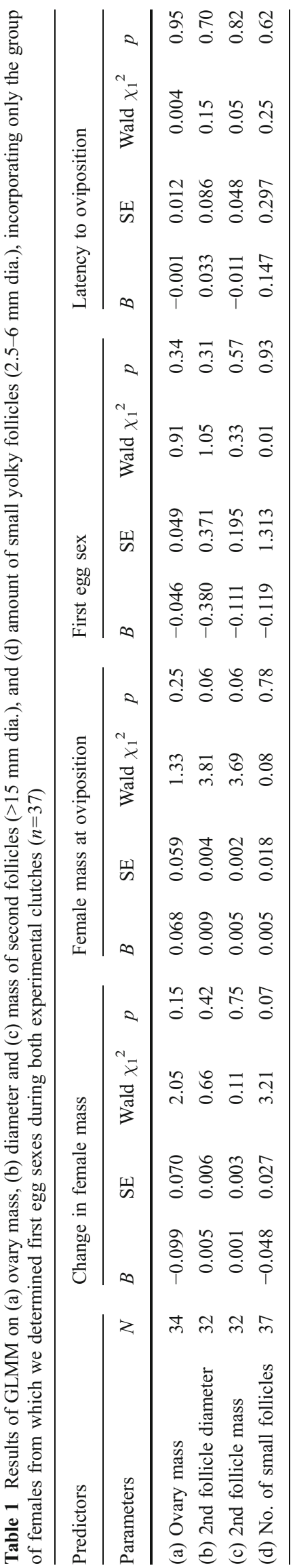

postovulatory follicle was present, and no further signs of follicle atresia were visible (Fig. 4). The birds were not related, and we could not find any other similarities between these two individuals that would have explained the occurrence of a third follicle. One of these two females had laid an unfertile first egg. Both females were 1 year of age, but this age class was over-represented in our study (17 females). Body mass of both females (423.7 and $523.6 \mathrm{~g}$ ) fell within the range of our experimental group (mean $460.3 \pm 38.0 \mathrm{SD}$, range 385.7 to $557.6 \mathrm{~g}$ ).

Two different females produced an unusual undersized second follicle (Figs. 2 and 3). One of these two females had laid an unfertile first egg, and the flaccid intermediate follicle ( $7.7 \mathrm{~mm}$ diam.) was of uneven shape, not entirely filled with yolk -although the follicular walls were not ruptured - and it was likely to be atretic. The other female had produced a fertile first egg, and the 11.0-mm diameter follicle seemed healthy (yellow, of even round shape, and vascularized), but clearly far smaller than a mature second follicle (Figs. 2 and 3). Again, in both females only one postovulatory follicle was present, and signs of additional follicle ripening or regression were absent. None of the experimental females had produced more than two eggs during the first trial clutch. Overall, in 50 out of 56 females we found the ovarian follicles to resemble the scenario depicted in Fig. 1a.

\section{Discussion}

The aim of this study was to test whether female pigeons manipulate their primary sex ratio via abortion of mature, preovulatory follicles of the un-preferred sex. This mechanism has been suggested to generally occur in bird species that produce small clutches or restrict biases in primary sex ratios to first eggs, and particularly in homing pigeons (Emlen 1997; Pike 2005). In the current experiment we showed in a subgroup of females, from which both first eggs from successive clutches developed embryos, an overall effect of change in body mass on change of embryo sex of first eggs (Goerlich et al. unpublished data) but we found no indication that the observed skew was brought about by follicle abortion and more than two follicles entering the phase of rapid yolk deposition. Finally we found no indication that latency to oviposition or sex of the first egg predicted ovarian or follicle parameters.

Also when including all experimental females we did not detect any support for the selective abortion theory since, directly after oviposition of the first egg, we found in only two out of 56 females two mature follicles $(>18.8 \mathrm{~mm}$ diameter) plus one postovulatory follicle instead of the expected one pre- and one postovulatory follicle. Neither of 


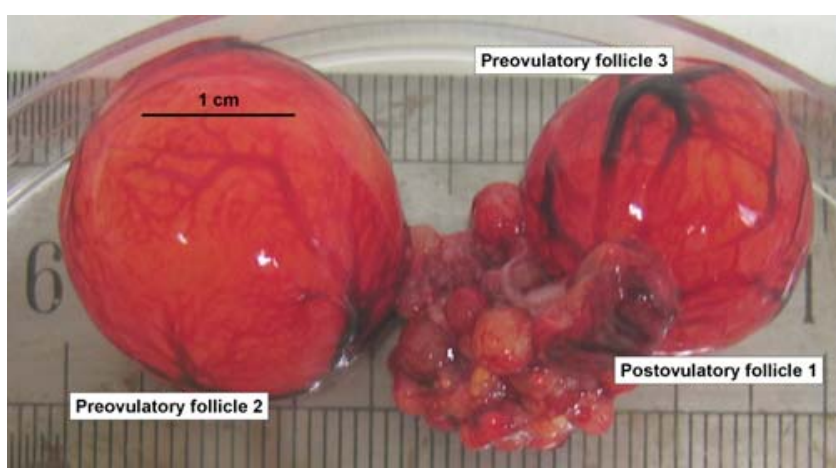

Fig. 4 One example of the ovaries $(N=2)$ that contained two mature follicles of preovulatory size plus one postovulatory follicle

these females differed from the rest of the experimental group with respect to weight and age, and only one of them produced a fertile first egg. Including autopsies of female pigeons used in our further sex ratio studies we recorded a third follicle only in six out of 192 individuals (3.1\%; Goerlich et al. unpublished data); thus the ovarian morphology described in Fig. 1a is the most common, and our results confirm the low frequencies of third follicles observed in domesticated pigeons before (Bartelmez 1912). Without sufficient replacement follicles, the hypothetical abortion of a preovulatory follicle would inevitably be accompanied by the negative effects described by Emlen (1997) such as prolonged egg intervals and reduced clutch sizes. However, both these effects have not been observed in studies manipulating primary sex ratio in pigeons (Pike 2005; Goerlich et al. 2009; Müller et al. unpublished data).

In two females out of the 56 experimental birds, the largest follicle we observed was not mature, but of intermediate size (one likely atretic). However, our expectations on follicle abortion were that females develop an additional replacement follicle, which then would be of intermediate size, either due to regression (Fig. 1c) or due to suppressed maturation (Fig. 1d). In contrast, these two females did not develop a mature second follicle, which suggests that the intermediate one resulted from a defect in development and was not destined to be ovulated.

Our predictions did not consider the recruitment of a third follicle into the rapid growth phase after the abortion of the first follicle since this would result in either of the following three scenarios, none of which receives substantial support in the literature. First, the third follicle would need to undergo the whole phase of rapid yolk deposition only starting after abortion of the previous mature follicle, which consequently would result in a delayed ovulation and enlarged laying gaps between egg 1 and 2. However, as stated above, this was not recorded in any of the studies showing primary sex ratio manipulation in pigeons (Pike 2005; Goerlich et al. 2009; Müller et al. unpublished data). Alternatively, the third follicle could be ovulated prema- turely, which would result in a considerably smaller and lighter yolk of second eggs, which was not the case in two of the pigeon studies (Goerlich et al. 2009; Müller et al. unpublished data; but see Pike 2005). Furthermore, since yolk resources are crucial for embryo development, ovulation of a premature follicle might jeopardize offspring viability (VanHeel et al. 1981). Finally, the third follicle could accelerate its growth to catch up on the lag in development (differential follicle growth; Badyaev et al. 2005). Dynamics of yolk deposition and follicle growth can be made visible by staining and measuring yolk layers (Grau 1976; Badyaev et al. 2005, 2008). When applying these methods in pigeons, Birrenkott et al. (1988) did not detect differences in the amount or thickness of yolk layers of first and second eggs within a clutch, which indicates that both follicles undergo the same time span and pattern of yolk deposition. However, it is not known whether these females were biasing the sex ratio of their clutch.

It is nevertheless possible that differential follicle growth prevents laying gaps caused by abortion of intermediate follicles, if growth curves of several oocytes overlap considerably, as is the case in passerines laying large clutches, like the house finch (Carpodacus mexicanus; Young and Badyaev 2004; Badyaev et al. 2008). Still, whether in pigeons follicles could catch up on a lag in development of 2 days (the usual ovulation interval between two follicles of ca. $44 \mathrm{~h}$ ) is doubtful.

\section{Conclusion}

If selective follicle abortion was to be the mechanism underlying primary sex ratio manipulation in pigeons, we should have seen aborted or regressed follicles and a large number of third follicles as potential replacement (Figs. 1b-d). However, although females of the current experiment adjusted the embryo sex of first eggs according to change in body mass, we did not find supporting evidence for the selective follicle abortion hypothesis in any of these birds. Although ovarian morphology of the majority of females resembled the scenario described in Fig. 1a, we did record two exceptional cases (out of 56) in which females developed three mature follicles, but clearly, the low frequency of this phenomenon does not support the potential for follicle abortion.

Therefore we should consider other possible mechanisms which might enable the mother to adjust primary sex ratio. A promising strategy would be to influence chromosome segregation during meiosis I in such a way that the preferred sex chromosome will be retained in the developing gamete. Meiotic drive has been proposed by several researchers and offers potential for hormones to interfere (Howe 1977; Komdeur et al. 2002; Correa et al. 2005; Rutkowska and 
Badyaev 2008). Another interesting approach to further explore follicle growth dynamics would be to carry on the work of Birrenkott et al. (1988) and investigate yolk deposition patterns in more detail. Although he did not find differences in between yolks, incorporating embryo sex might yield a different picture (Badyaev et al. 2005).

It is certainly worthwhile to explore these potential mechanisms in the homing pigeon, a species repeatedly showing biases in primary offspring sex ratio in response to different experimental treatments (Goerlich et al. 2009; unpublished data; Müller et al. unpublished data; Pike 2005).

Acknowledgments Many thanks go to Marcel Koen and Maite Eikelenboom who helped with the experimental procedures.

Open Access This article is distributed under the terms of the Creative Commons Attribution Noncommercial License which permits any noncommercial use, distribution, and reproduction in any medium, provided the original author(s) and source are credited.

\section{References}

Alonso-Alvarez C (2006) Manipulation of primary sex-ratio: an updated review. Avian Poult Biol Rev 17:1-20

Badyaev AV, Schwabl H, Young RL, Duckworth RA, Navara KJ, Parlow $\mathrm{AF}$ (2005) Adaptive sex differences in growth of pre-ovulation oocytes in a passerine bird. Proc R Soc Lond B Biol Sci 272:2165-2172

Badyaev AV, Young RL, Hill GE, Duckworth RA (2008) Evolution of sex-biased maternal effects in birds. IV. Intra-ovarian growth dynamics can link sex determination and sex-specific acquisition of resources. J Evol Biol 21:449-460

Bartelmez GW (1912) The bilaterality of the pigeon's egg. I. A study in egg organization from the first growth period of the oocyte to the beginning of cleavage. J Morphol 23:269-328

Beukeboom L, Dijkstra C, Daan S, Meijer T (1988) Seasonality of clutch size determination in the kestrel Falco tinnunculus: an experimental approach. Ornis Scand 19:41-48

Birrenkott GP, Shoop MA, Cooper K, Wiggins M (1988) Ovarian follicular-growth and maturation in the domestic pigeon and guinea fowl (Numida-Meleagris). Poult Sci 67:1783-1786

Blockstein DE (1989) Crop milk and clutch size in mourning doves. Wilson Bull 101:11-25

Correa SM, Adkins-Regan E, Johnson PA (2005) High progesterone during avian meiosis biases sex ratios toward females. Biol Lett $1: 215-218$

Cuthbert NL (1945) The ovarian cycle of the ring dove (Streptopelia Risoria). J Morphol 77:351-377

Emlen ST (1997) When mothers prefer daughters over sons. Trends Ecol Evol 12:291-292
Fazeli A (2008) Maternal communication with gametes and embryos. Theriogenology 70:1182-1187

Georgiou AS, Snijders APL, Sostaric E, Aflatoonian R, Vazquez JL, Vazquez JM, Roca J, Martinez EA, Wright PC, Fazeli A (2007) Modulation of the oviductal environment by gametes. J Proteome Res 6:4656-4666

Gilbert AB, Perry MM, Waddington D, Hardie MA (1983) Role of atresia in establishing the follicular hierarchy in the ovary of the domestic hen (Gallus domesticus). J Reprod Fertil 69:221-227

Goerlich VC, Dijkstra C, Schaafsma SM, Groothuis TGG (2009) Testosterone has a long term effect on primary sex ratio of first eggs in pigeons - in search of a mechanism. Gen Comp Endocrinol 163(1-2):184-192

Grafen A, Hails R (2002) Modern statistics for the life sciences. Oxford University Press, Oxford

Grau CR (1976) Ring structure of avian egg-yolk. Poult Sci 55:14181422

Gutierrez RJ, Braun CE, Zapatka TP (1975) Reproductive biology of band-tailed pigeon in Colorado and New-Mexico. Auk 92:665677

Haywood S (1993) Sensory and hormonal-control of clutch size in birds. Q Rev Biol 68:33-60

Howe HF (1977) Sex-ratio adjustment in common grackle. Science 198:744-746

Johnson AL (2000) Reproduction in the female. In: Whittow GC (ed) Sturkie's Avian Physiology, 5th edn. Academic, San Diego, pp 569-596

Komdeur J, Magrath MJL, Krackow S (2002) Pre-ovulation control of hatchling sex ratio in the Seychelles warbler. Proc R Soc B 269:1067-1072

Krackow S (1995) Potential mechanisms for sex-ratio adjustment in mammals and birds. Biol Rev Camb Philos Soc 70:225-241

Levi WM (1945) The pigeon. Bryan, Columbia

March GL, Sadleir RMFS (1970) Studies on band-tailed pigeon (Columba Fasciata) in British-Columbia 1. Seasonal changes in gonadal development and crop gland activity. Can J Zool 48:1353-1357

Nager RG, Monaghan P, Houston DC (2001) The cost of egg production: increased egg production reduces future fitness in gulls. J Avian Biol 32:159-166

Pike TW (2005) Sex ratio manipulation in response to maternal condition in pigeons: evidence for pre-ovulatory follicle selection. Behav Ecol Sociobiol 58:407-413

Pike TW, Petrie M (2003) Potential mechanisms of avian sex manipulation. Biol Rev 78:553-574

Romanoff AL, Romanoff AJ (1949) The avian egg. Wiley, New York

Rutkowska J, Badyaev AV (2008) Meiotic drive and sex determination: molecular and cytological mechanisms of sex ratio adjustment in birds. Philos Trans R Soc Lond B Biol Sci 363:1675-1686

VanHeel B, Vandeputtepoma J, Desmeth M (1981) Resorption of yolk lipids by the pigeon embryo. Comp Biochem Physiol A Comp Physiol 68:641-646

Young RL, Badyaev AV (2004) Evolution of sex-biased maternal effects in birds. I. Sex-specific resource allocation among simultaneously growing oocytes. J Evol Biol 17:1355-1366 